\title{
Expression profiling based on coexpressed modules in obese prepubertal children
}

\author{
J.F. Zhang', G.Y. Yao ${ }^{1}$ and Y.H. Wu ${ }^{2}$ \\ ${ }^{1}$ Department of Child Health Care, Shanghai Children's Hospital, \\ Shanghai Jiao Tong University, Shanghai, China \\ ${ }^{2}$ Department of Pediatrics, Zhabei District Central Hospital, Shanghai, China \\ Corresponding author: Y.H. Wu \\ E-mail: xjzjfgoda@sina.com
}

Genet. Mol. Res. 11 (3): 3077-3085 (2012)

Received January 5, 2012

Accepted June 22, 2012

Published August 30, 2012

DOI http://dx.doi.org/10.4238/2012.August.31.5

\begin{abstract}
The aim of this study was to identify related genes and the underlying molecular mechanisms in obese patients who show a series of clinical and metabolic abnormalities known as metabolic syndrome. We identified expression profiles through a coexpression network. In addition, a similarity matrix and expression modules were constructed based on domain and pathway enrichment analysis. The genes in module 1 were mainly involved in the metabolism of xenobiotics by cytochrome $\mathrm{P} 450$, aldosterone-regulated sodium reabsorption, and focal adhesion owing to the presence of aldo/ketoreductase, basic helix-loop-helix, von Willebrand factor, Frizzled-related domain, and other domains. The genes in module 3 may be involved in cell cycle (hsa04110) and DNA replication (hsa03030) pathways through minichromosome maintenance, serine/threonine protein kinase, the protein kinase domain, and other domains. We analyzed the published molecular mechanisms of obesity and found many genes and pathways that have not been given enough attention and require further confirmation.
\end{abstract}

Keys words: Obesity; Coexpression network; Cell cycle; Pathway enrichment analysis 


\section{INTRODUCTION}

The prevalence of obesity in children in developed and developing countries has increased dramatically in recent decades. Obese patients typically display a series of clinical and metabolic abnormalities known as metabolic syndrome, characterized by insulin resistance (IR), hyperglycemia, high very-low-density lipoprotein triacylglycerols, low- and high-density lipoprotein cholesterol, and hypertension (Grundy, 2004; Olza et al., 2011).

In response to these metabolic abnormalities, leptin concentrations in plasma and serum significantly increase in obese children, and the levels are directly proportional to the severity of adiposity. Leptin regulates body weight mainly through its anorectic effect on the hypothalamus. In addition, this hormone also has effects, such as inflammatory response, on the immune system. In prepubertal obese children, oxidative stress increases malondialdehyde, and homocysteine levels are well correlated with serum leptin level (Martos et al., 2006). Serum interleukin (IL)-1 $\beta$, IL-6, and tumor necrosis factor $\alpha$ levels are also significantly higher in obese children than those in controls. In contrast, antioxidant activities of superoxide dismutase, glutathione peroxidase, and IL-2 are decreased (Aygun et al., 2005; Martos et al., 2006; Ustundag et al., 2007; Olza et al., 2010). However, high leptin levels in childhood obesity do not seem to be associated with altered complete blood count parameters or increased peripheral CD34(+) progenitor cell count (Kinik et al., 2005).

Emerging data have demonstrated that insulin-like growth factor-binding protein-2 (IGFBP-2) expression may potentially be one of the local mechanisms used by adipocytes to limit further fat gain. Low circulating IGFBP-2 is associated with adiposity and reduced insulin sensitivity (Heald et al., 2006). Transgenic mice overexpressing IGFBP-2 in adipocytes do not develop obesity and IR, even when overfed (Wheatcroft et al., 2007). Circulating IGFBP-2 is positively associated with insulin sensitivity in prepubertal obese children, and IGFBP-2 expression is associated with fat mass percentage, insulin sensitivity, free insulin-like growth factor-I, and leptin (Claudio et al., 2010).

In obese children, obesity and IR are associated with higher levels of circulating endothelial dysfunction biomarkers, such as soluble intercellular adhesion molecule-1, soluble vascular cell adhesion molecule-1 (sVCAM-1), leukocyte adhesion molecule-1, von Willebrand factor (vWF), C-reactive protein (CRP), plasminogen activator inhibitor-1, and leptin. Obese children display significantly elevated soluble intercellular adhesion molecule-1, vWF, insulin, homeostasis model assessment for IR, CRP, plasminogen activator inhibitor-1, and leptin values (Valle et al., 2007). sVCAM-1 levels are also significantly increased in obese children after oral glucose tolerance testing, and sVCAM-1 is positively correlated with insulin and homeostasis model assessment values. Fasting CRP is positively correlated with body mass index and low-density lipoproteins, whereas leukocyte adhesion molecule-1 is positively correlated with total cholesterol (Süheyl et al., 2005).

Obesity is associated with a high incidence of abnormal serum levels of alanine aminotransferase, and elevation of alanine aminotransferase in obese children is a marker for nonalcoholic fatty liver disease, which is caused in part by IR (Calcaterra et al., 2011). Elevated plasma uric acid levels are associated with obesity and could be an expression of an insulinresistant state (Gil-Campos et al., 2009). Increased concentrations of 16:1n-7 and decreased proportions of 20:4n-6 and 22:5n-6 in plasma lipids have been predicted as early markers of 
metabolic syndrome in prepubertal children (Gil-Campos et al., 2008).

However, additional genes and their corresponding mechanisms are still essential to explore. In this study, we used a coexpression strategy to identify new genes and their underlying mechanisms. One method of identifying coexpressed modules is through the construction of a similarity matrix derived from a collection of microarray data sets. Distance in the similarity matrix is determined with a correlation metric (e.g.; Pearson's r), in which a comprehensive $\mathrm{m} \times \mathrm{m}$ matrix of correlation values is generated that represents expression similarity. The similarity matrix is then transformed into an "adjacency matrix" representing an undirected graph in which edges (coexpression) exist between 2 nodes when a correlation value in the matrix is above a significance threshold. A weighted gene coexpression network analysis (WGCNA) (Langfelder and Horvath, 2008) is then applied to circumscribe groups of network nodes that are highly connected. Genes in these modules have been shown to participate in similar biological processes; therefore, WGCNA packages can be applied to module genes with no known function that are connected to module genes of known function. We anticipate that our study will lay a theoretical foundation for further experimental investigation.

\section{MATERIAL AND METHODS}

\section{Microarray data analysis and coexpression network construction}

Analysis was performed in the omental (OM) adipose of obese prepubertal children. Obese children display IR and other metabolic abnormalities at higher rates than those in normal-weight children. The results provide insight into the molecular mechanisms underlying the pathogenesis of childhood obesity using the microarray data GSE9624, which was downloaded by Gene Expression Omnibus (http://www.ncbi.nlm.nih.gov/geo). Adipose tissues and blood samples were obtained from 11 children -5 obese (body mass index adjusted for age and gender $\mathrm{z}$ score $>2$ ) and 6 nonobese - undergoing appendix surgery in Unit of Pediatric Endocrinology, Reina Sofía University Hospital. Approximately $400 \mathrm{mg}$ adipose tissue was taken and immediately immersed in RNAlater solution (QIAGEN, Hilden, Germany) and stored at $-80^{\circ} \mathrm{C}$ for gene expression analysis. Informed consent was obtained from all patients and their parents after the nature of the study was explained, and the experimental design was approved from an ethical and scientific standpoint by the Ethics Committee responsible for research at the hospital.

To construct the expression profiles, we selected the differentially expressed genes (DEGs) using the limma method (Diboun et al., 2006) with moderated $t$-statistics with empirical Bayes shrinkage of the standard errors. We selected DEGs with $\mathrm{P}$ values $<0.05$ and foldchange values $>2$.

A similarity matrix was then constructed by performing pairwise Pearson's correlations for every probe set across all samples. Next, the WGCNA package (Langfelder and Horvath, 2008) was used to convert the similarity matrix into an adjacency matrix by raising the similarity matrix to a power of 6 . The power chosen was the one that best approximated scale-free behavior in the resulting network and was selected by the software (Ficklin and Feltus, 2011). To combine the various modules, we constructed a coexpression network with a Pearson's correlation coefficient (PCC) $>0.75$ for any 2 DEGs. 


\section{Functional enrichment analysis}

For this study, modules were determined using the WGCNA method that groups nodes by measures of similarity. InterPro (Apweiler et al., 2001) and Kyoto Encyclopedia of Genes and Genomes (Kanehisa et al., 2008) terms were used for functional annotation of these gene modules. The online DAVID (the database for annotation, visualization and integrated discovery) tool (Dennis et al., 2003) was used to perform functional enrichment using hypergeometric distribution against each network module and the genome background.

\section{RESULTS}

\section{Expression profiling selection and module detection}

After chip analysis, 342 DEGs with fold-change values $>2$ and $\mathrm{P}$ values $<0.05$ were selected. To obtain the coexpression value of any 2 DEGs, the similarity matrix was constructed with the calculated PCC (Brown et al., 1962). Then, the WGCNA packages were used to find the modules. Six modules marked by various colors were detected (Figure 1). The coexpression networks were also analyzed with the PCC $>0.75$. Then, we drew the network in which that the modules were marked with various colors (Figure 2).

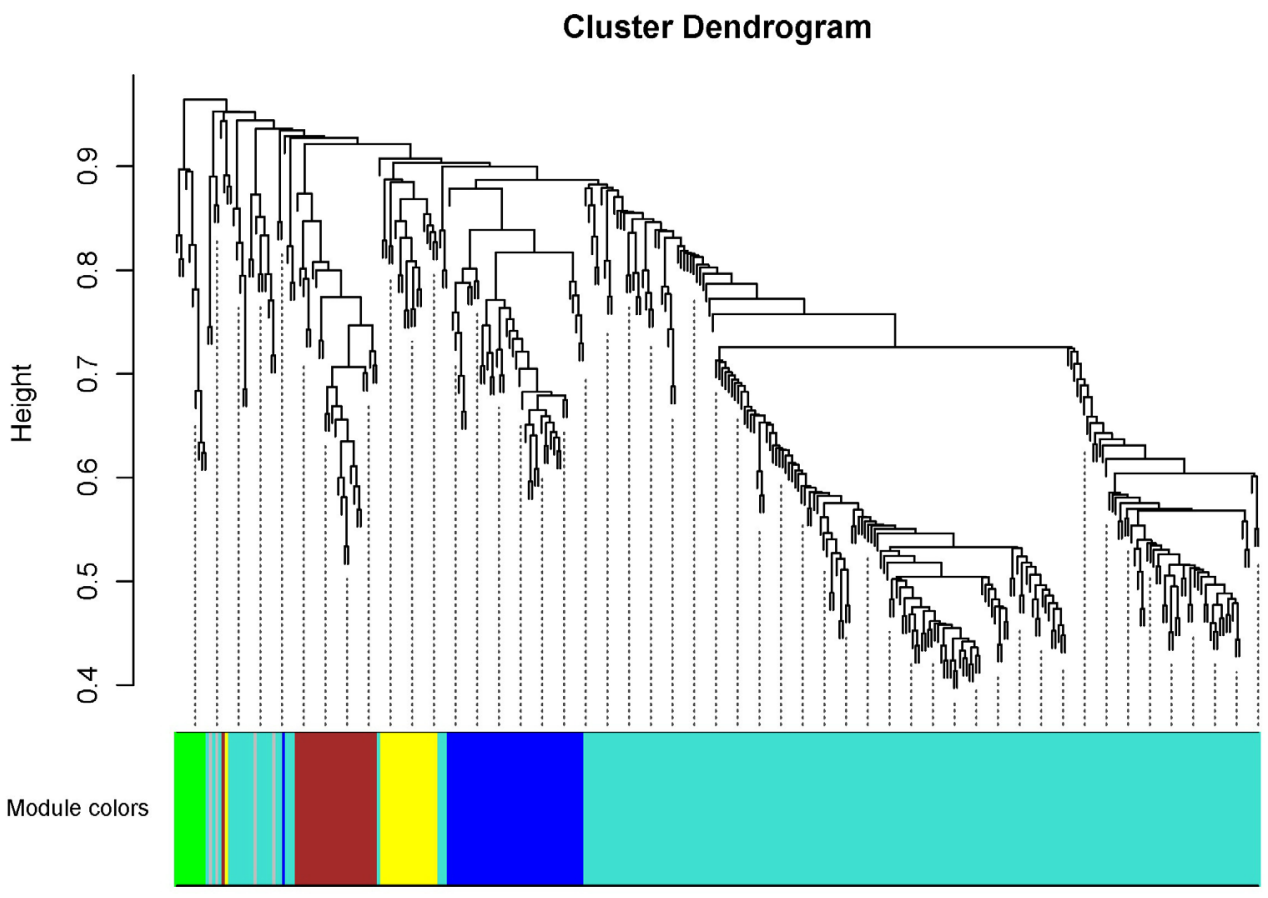

Figure 1. Hierarchical clustering dendrogram with the weighted gene coexpression network analysis. The color band underneath the tree shows the results of defining modules as branches of the tree. 'Gray' (module 0) is reserved for unassigned genes. 

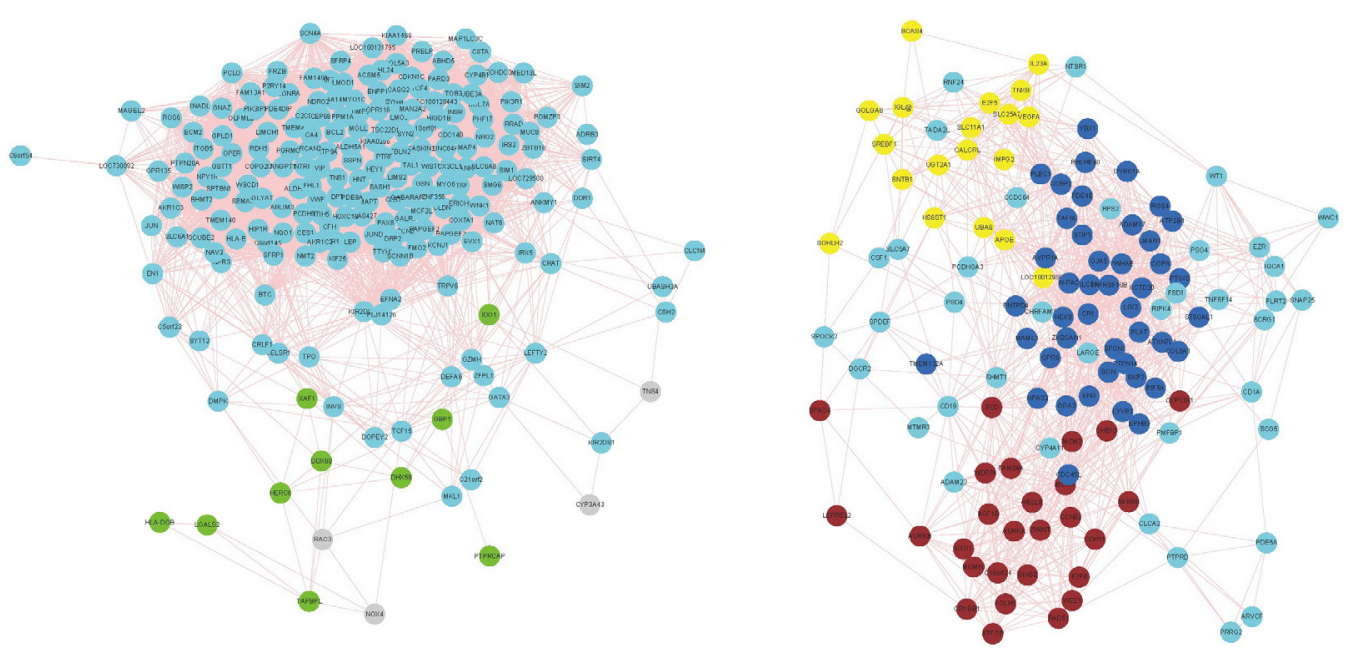

Figure 2. The coexpression network marked by various colors for different modules. Gray color indicates the modules 0 for unassigned genes; turquoise indicates module 1 ; blue indicates module 2; brown indicates module 3; yellow indicates module 4; green indicates module 5; node indicates the differentially expressed genes; edge indicates the Pearson's correlation coefficient $>0.75$ between any two nodes.

\section{Functional enrichment analysis}

A functional enrichment analysis was performed to examine the modules. The DAVID program was used to find the enriched Gene Ontology terms. Domains of various modules were analyzed to find the potential function. Table 1 displays the domain information of 6 modules. The basic helix-loop-helix (bHLH) dimerization region (IPR001092) was enriched in both module 1 and module 4 . Through the pathway enrichment analysis, we found that module 1 was enriched in aldosterone-regulated sodium reabsorption (hsa04960), metabolism of xenobiotics by cytochrome P450 (hsa00980), and focal adhesion (hsa04510); module 3 was enriched in cell cycle (hsa04110) and DNA replication (hsa03030; Table 2).

\section{DISCUSSION}

Our results showed that 342 DEGs were screened to construct the coexpression network. Six modules were detected (marked by various colors; see Figure 1). Furthermore, the underlying mechanism of these 6 modules was predicted by domain and pathway enrichment analyses. Our results revealed consistency between these 2 functional analysis methods. The genes in module 1 were mainly involved in metabolism of xenobiotics by cytochrome P450, aldosterone-regulated sodium reabsorption, and focal adhesion pathways owing to the presence of aldo/ketoreductase (AKR), bHLH, vWF, Frizzledrelated domain, and other domains. We discuss the corresponding genes according to the results of previous reports. 


\begin{tabular}{|c|c|c|c|}
\hline Category & Term (domain) & Count & $P$ value \\
\hline Module 0 & NA & NA & NA \\
\hline \multirow[t]{29}{*}{ Module 1} & IPR001092:Basic helix-loop-helix dimerisation region bHLH & 8 & $6.38 \mathrm{E}-04$ \\
\hline & IPR001134:Netrin domain & 4 & 0.002982 \\
\hline & IPR018170:Aldo/ketoreductase, conserved site & 3 & 0.00822 \\
\hline & IPR002011:Receptor tyrosine kinase, class II, conserved site & 3 & 0.011464 \\
\hline & IPR001781:Zinc finger, LIM-type & 5 & 0.014012 \\
\hline & IPR001007:von Willebrand factor, type C & 4 & 0.014205 \\
\hline & IPR001395:Aldo/ketoreductase & 3 & 0.019333 \\
\hline & IPR018933:Netrin module, non-TIMP type & 3 & 0.019333 \\
\hline & IPR015526:Frizzled related & 3 & 0.019333 \\
\hline & IPR013655:PAS fold-3 & 3 & 0.02157 \\
\hline & IPR011598:Helix-loop-helix DNA-binding & 5 & 0.022317 \\
\hline & IPR000048:IQ calmodulin-binding region & 5 & 0.025006 \\
\hline & IPR010578:Single-minded, C-terminal & 2 & 0.025291 \\
\hline & IPR000024:Frizzled cysteine-rich domain & 3 & 0.026345 \\
\hline & IPR002017:Spectrin repeat & 3 & 0.028878 \\
\hline & IPR001084:Tubulin-binding Tau protein & 2 & 0.037696 \\
\hline & IPR002112:Transcription factor Jun & 2 & 0.037696 \\
\hline & IPR005643:Jun-like transcription factor & 2 & 0.037696 \\
\hline & IPR000742:EGF-like, type 3 & 7 & 0.038149 \\
\hline & IPR013767:PAS-fold & 3 & 0.039913 \\
\hline & IPR001610:PAC motif & 3 & 0.042885 \\
\hline & IPR006210:EGF-like & 7 & 0.043847 \\
\hline & IPR016181:Acyl-CoA N-acyltransferase & 3 & 0.05889 \\
\hline & IPR018159:Spectrin/alpha-actinin & 3 & 0.05889 \\
\hline & IPR000014:PAS & 3 & 0.065783 \\
\hline & IPR002035:von Willebrand factor, type A & 4 & 0.079662 \\
\hline & IPR013032:EGF-like region, conserved site & 8 & 0.081311 \\
\hline & IPR004241:Light chain 3 (LC3) & 2 & 0.085766 \\
\hline & IPR001605:Spectrin/pleckstrin-like & 2 & 0.085766 \\
\hline Module 2 & NA & NA & NA \\
\hline \multirow[t]{8}{*}{ Module 3} & IPR018525:DNA-dependent ATPase MCM, conserved site & 2 & 0.01147 \\
\hline & IPR001208:DNA-dependent ATPase MCM & 2 & 0.012895 \\
\hline & IPR008271:Serine/threonine protein kinase, active site & 4 & 0.013833 \\
\hline & IPR017442:Serine/threonine protein kinase-related & 4 & 0.014361 \\
\hline & IPR017441:Protein kinase, ATP binding site & 4 & 0.026766 \\
\hline & IPR000719:Protein kinase, core & 4 & 0.030057 \\
\hline & IPR002290:Serine/threonine protein kinase & 3 & 0.0531 \\
\hline & IPR012340:Nucleic acid-binding, OB-fold & 2 & 0.073675 \\
\hline Module 4 & IPR001092:Basic helix-loop-helix dimerisation region bHLH & 2 & 0.097912 \\
\hline \multirow[t]{6}{*}{ Module 5} & IPR011545:DNA/RNA helicase, DEAD/DEAH box type, $N$-terminal & 2 & 0.032484 \\
\hline & IPR003597:Immunoglobulin C1-set & 2 & 0.036151 \\
\hline & IPR003006:Immunoglobulin/major histocompatibility complex, conserved site & 2 & 0.046042 \\
\hline & IPR001650:DNA/RNA helicase, C-terminal & 2 & 0.056357 \\
\hline & IPR014021:Helicase, superfamily 1 and 2, ATP-binding & 2 & 0.056357 \\
\hline & IPR014001:DEAD-like helicase, N-terminal & 2 & 0.057383 \\
\hline
\end{tabular}

$\mathrm{NA}=$ not available.

Regarding the metabolism of xenobiotics by cytochrome P450 pathway, AKR1C enzymes, especially AKR1C2 and AKR1C3, are highly expressed and activated in subcutaneous and $\mathrm{OM}$ adipose tissue. This activation could reverse the inhibitory effect of androgen dihydrotestosterone on adipocyte differentiation, ultimately leading to obesity (Wake et al., 2007; Blouin et al., 2009). AKR1C activity and messenger RNA (mRNA) have also been detected in subcutaneous and OM adipose tissue in women, and OM AKR1C of progesterone is highest in women with visceral obesity. Significant positive correlations are also found between OM AKR1C activity and OM adipocyte diameter and OM adipose tissue lipoprotein lipase activity (Blouin et al., 2005). 
Table 2. Pathway enrichment analysis.

\begin{tabular}{llcc}
\hline Category & Term (pathway) & Count & P value \\
\hline Module 0 & NA & NA & NA \\
Module 1 & hsa04960:Aldosterone-regulated sodium reabsorption & 5 & 0.003867 \\
& hsa00980:Metabolism of xenobiotics by cytochrome P450 & 4 & 0.069765 \\
Module 2 & hsa04510:Focal adhesion & 7 & 0.099776 \\
Module 3 & NA & NA & NA \\
& hsa04110:Cell cycle & 5 & 6.52 E-05 \\
Module 4 & hsa03030:DNA replication & 2 & 0.068642 \\
Module 5 & NA & NA & NA \\
\hline
\end{tabular}

NA $=$ not available.

The aldosterone-regulated sodium reabsorption pathway shows enrichment with the insulin receptor (INSR), insulin receptor substrate 2 (IRS-2), and phosphatidylinositol 3-kinase (PI3K) R1 genes. Muscle-specific INSR knockout mice (fat-specific disruption of the INSR gene) exhibit a muscle-specific reduction in receptor content and early signaling events (Bruning et al., 1998). These mice also display low fat mass, loss of the normal relationship between plasma leptin and body weight, protection against age-related and hypothalamic lesion-induced obesity, and obesity-related glucose intolerance. Fat-specific INSR knockout mice also exhibit polarization of large and small cells, which differ in their expression of fatty acid synthase, CCAAT/enhancer-binding protein $\alpha$, and sterol regulatory element-binding protein 1 . In brief, INSRs in adipocytes are critical for the development of obesity (Blüher et al., 2002).

Knockout mice lacking IRS-2 show IR with abnormal glucose tolerance at birth and progressively develop fasting hyperglycemia as a result of inadequate compensatory insulin secretion owing to reduced $\beta$-cell mass. More recently, IRS-2-defective female mice have been shown to exhibit defects in the hypothalamic-pituitary-ovarian axis resulting in infertility and moderate obesity (Sesti et al., 2001). IRS-2-defective females also show increased food intake and increased body fat despite elevated leptin levels. These results raise the intriguing possibility that dysregulation of IRS-2 expression or function may be a molecular defect responsible for insulin-resistant conditions (Masaki et al., 2004).

PI3K couples the leptin and insulin signaling pathways via the insulin receptor substrates IRS-1 and IRS-2. Hence, defective activation of PI3K could be a novel mechanism of peripheral leptin or IR. The association of the PI3KR1 SNPrs1550805 with serum leptin and body fat may reflect a diminished capability of PI3K to signal via IRS-1 or IRS-2 in response to leptin (Jamshidi et al., 2006).

The focal adhesion pathway shows enrichment of B-cell lymphoma 2 (BCL-2) and collagen alpha-3(V) (COL5A3). Obesity is linked to functional brown adipose tissue atrophy, partially owing to adipocyte apoptosis. The Bcl-2/Bcl-2-associated X protein mRNA and protein ratios of brown adipocytes of obese rats are lower than those of their lean littermates (Briscini et al., 1998). Col5a3 knockout mice, which lack the a3(V) chain, can exhibit gender-specific differences in adipose deposition. Female Col5a 3 knockout mice display reduced thickness of hypodermal adipose deposits as well as resistance to diet-induced obesity compared with those of controls (Butz, 2010).

In addition, secreted Frizzled-related protein 1 (SFRP1) is an endogenous modulator of $\mathrm{Wnt} / \beta$-catenin signaling and participates in the paracrine regulation of human adipogenesis. SFRP1 is preferentially expressed in mature adipocytes in human adipose tissue. SFRP1 ex- 
pression in human tissues peaks in patients with mild obesity but gradually falls in morbidly obese subjects. This knock-on effect prevents further adipose tissue expansion and the development of metabolic complications in these individuals (Lagathu et al., 2010).

The genes in module 3 may be involved in cell cycle (hsa04110) and DNA replication (hsa03030) pathways through mini-chromosome maintenance (MCM), serine/threonine protein kinase, protein kinase domain, and other domains. DNA replication initiation in adipocytes requires the assembly of a pre-replication complex, including origin recognition complexes 1-6, cell division cycle 6, chromatin licensing and DNA replication factor 1, human acetylase binding to Orc1, and MCM 2-7 at origins of DNA replication (Johmura et al., 2008).

$\beta$-cell mass expansion is one mechanism through which obese animals compensate for IR and prevent diabetes. Forkhead box protein M1 is a transcription factor that can regulate the expression of multiple cell cycle genes and is necessary for the maintenance of adult $\beta$-cell mass, $\beta$-cell proliferation, and glucosehomeostasis. G1/S transition regulator cyclin e2 is positively correlated with forkhead box protein M1 mRNA levels (Davis et al., 2010). Insulin receptor tyrosine kinase activity is defective in the skeletal muscle of insulin-resistant obese mice (Le Marchand-Brustel et al., 1985). This defect may result from the increased phosphorylation on serine/threonine residues by protein kinase $\mathrm{C}$. Therefore, protein kinase $\mathrm{C}$ $\beta$ content in the muscle tissue of obese patients is significantly higher than that in the muscles of lean subjects (Itani et al., 2000).

In summary, we constructed 6 coexpression modules and found that the genes in module 1 were mainly involved in metabolism of xenobiotics by cytochrome P450, aldosteroneregulated sodium reabsorption, and focal adhesion owing to the presence of AKR, bHLH, vWF, Frizzled-related domain, and other domains. The genes in module 3 may be involved in cell cycle (hsa04110) and DNA replication (hsa03030) pathways through MCM, serine/threonine protein kinase, protein kinase domain, and other domains. Further research should focus on confirming unidentified genes and exploring their influence on obesity.

\section{REFERENCES}

Apweiler R, Attwood TK, Bairoch A, Bateman A, et al. (2001). The InterPro database, an integrated documentation resource for protein families, domains and functional sites. Nucleic Acids Res. 29: 37-40.

Aygun AD, Gungor S, Ustundag B, Gurgoze MK, et al. (2005). Proinflammatory cytokines and leptin are increased in serum of prepubertal obese children. Mediators Inflamm. 2005: 180-183.

Blouin K, Blanchette S, Richard C, Dupont P, et al. (2005). Expression and activity of steroid aldoketoreductases 1C in omental adipose tissue are positive correlates of adiposity in women. Am. J. Physiol. Endocrinol. Metab. 288: E398-E404.

Blouin K, Veilleux A, Luu-The V and Tchernof A (2009). Androgen metabolism in adipose tissue: recent advances. Mol. Cell. Endocrinol. 301: 97-103.

Blüher M, Michael MD, Peroni OD, Ueki K, et al. (2002). Adipose tissue selective insulin receptor knockout protects against obesity and obesity-related glucose intolerance. Dev. Cell 3: 25-38.

Briscini L, Tonello C, Dioni L, Carruba MO, et al. (1998). Bcl-2 and Bax are involved in the sympathetic protection of brown adipocytes from obesity-linked apoptosis. FEBS Lett. 431: 80-84.

Brown BW Jr, Lucero RJ and Foss AB (1962). A situation where the Pearson correlation coefficient leads to erroneous assessment of reliability. J. Clin. Psychol. 18: 95-97.

Bruning JC, Michael MD, Winnay JN, Hayashi T, et al. (1998). A muscle-specific insulin receptor knockout exhibits features of the metabolic syndrome of NIDDM without altering glucose tolerance. Mol. Cell 2: 559-569.

Butz DH (2010). Characterization of Novel Col5a3 Knockout and Tll1 Conditional Knockout Mice. Doctoral thesis, The University of Wisconsin, Madison.

Calcaterra V, Muratori T, Klersy C, Albertini R, et al. (2011). Early-onset metabolic syndrome in prepubertal obese children and the possible role of alanine aminotransferase as marker of metabolic syndrome. Ann. Nutr. Metab. 58: 307-314. 
Claudio M, Benjamim F, Riccardo B, Massimiliano C, et al. (2010). Adipocytes IGFBP-2 expression in prepubertal obese children. Obesity (Silver Spring) 18: 2055-2057.

Davis DB, Lavine JA, Suhonen JI, Krautkramer KA, et al. (2010). FoxM1 is up-regulated by obesity and stimulates betacell proliferation. Mol. Endocrinol. 24: 1822-1834.

Dennis G Jr, Sherman BT, Hosack DA, Yang J, et al. (2003). DAVID: Database for Annotation, Visualization, and Integrated Discovery. Genome Biol. 4: 3.

Diboun I, Wernisch L, Orengo CA and Koltzenburg M (2006). Microarray analysis after RNA amplification can detect pronounced differences in gene expression using limma. BMC Genomics 7: 252.

Ficklin SP and Feltus FA (2011). Gene coexpression network alignment and conservation of gene modules between two grass species: maize and rice. Plant Physiol. 156: 1244-1256.

Gil-Campos M, del Carmen Ramírez-Tortosa M, Larque E, Linde J, et al. (2008). Metabolic syndrome affects fatty acid composition of plasma lipids in obese prepubertal children. Lipids 43: 723-732.

Gil-Campos M, Aguilera CM, Cañete R and Gil A (2009). Uric acid is associated with features of insulin resistance syndrome in obese children at prepubertal stage. Nutr. Hosp. 24: 607-613.

Grundy SM (2004). Obesity, metabolic syndrome, and cardiovascular disease. J. Clin. Endocrinol. Metab. 89: 2595-2600.

Heald AH, Kaushal K, Siddals KW, Rudenski AS, et al. (2006). Insulin-like growth factor binding protein-2 (IGFBP-2) is a marker for the metabolic syndrome. Exp. Clin. Endocrinol. Diabetes 114: 371-376.

Itani SI, Zhou Q, Pories WJ, MacDonald KG, et al. (2000). Involvement of protein kinase C in human skeletal muscle insulin resistance and obesity. Diabetes 49: 1353-1358.

Jamshidi Y, Snieder H, Wang X, Pavitt MJ, et al. (2006). Phosphatidylinositol 3-kinase p85alpha regulatory subunit gene PIK3R1 haplotype is associated with body fat and serum leptin in a female twin population. Diabetologia 49: 2659-2667.

Johmura Y, Osada S, Nishizuka M and Imagawa M (2008). FAD24 acts in concert with histone acetyltransferase HBO1 to promote adipogenesis by controlling DNA replication. J. Biol. Chem. 283: 2265-2274.

Kanehisa M, Araki M, Goto S, Hattori M, et al. (2008). KEGG for linking genomes to life and the environment. Nucleic Acids Res. 36: D480-D484.

Kinik ST, Ozbek N, Yucel M, Haberal A, et al. (2005). Correlations among serum leptin levels, complete blood count parameters and peripheral CD34(+) cell count in prepubertal obese children. Ann. Hematol. 84: 605-608.

Lagathu C, Christodoulides C, Tan CY, Virtue S, et al. (2010). Secreted frizzled-related protein 1 regulates adipose tissue expansion and is dysregulated in severe obesity. Int. J. Obes. 34: 1695-1705.

Langfelder P and Horvath S (2008). WGCNA: an R package for weighted correlation network analysis. BMC Bioinformatics 9: 559.

Le Marchand-Brustel Y, Gremeaux T, Ballotti R and Van Obberghen E (1985). Insulin receptor tyrosine kinase is defective in skeletal muscle of insulin-resistant obese mice. Nature 315: 676-679.

Martos R, Valle M, Morales R, Cañete R, et al. (2006). Hyperhomocysteinemia correlates with insulin resistance and lowgrade systemic inflammation in obese prepubertal children. Metabolism 55: 72-77.

Masaki T, Chiba S, Noguchi H, Yasuda T, et al. (2004). Obesity in insulin receptor substrate-2-deficient mice: disrupted control of arcuate nucleus neuropeptides. Obes. Res. 12: 878-885.

Olza J, Gil-Campos M, Leis R, Aguilera CM, et al. (2010). Biomarkers of inflammation in prepubertal obese Spanish children. Proc. Nutr. Soc. 69: E233.

Olza J, Gil-Campos M, Leis R, Bueno G, et al. (2011). Presence of the metabolic syndrome in obese children at prepubertal age. Ann. Nutr. Metab. 58: 343-350.

Sesti G, Federici M, Hribal ML, Lauro D, et al. (2001). Defects of the insulin receptor substrate (IRS) system in human metabolic disorders. FASEB J. 15: 2099-2111.

Süheyl EF, Hasanoglu A, Tumer L, Ozbay F, et al. (2005). Endothelial activation and inflammation in prepubertal obese Turkish children. Metabolism 54: 1384-1389.

Ustundag B, Gungor S, Aygun AD, Turgut M, et al. (2007). Oxidative status and serum leptin levels in obese prepubertal children. Cell Biochem. Funct. 25: 479-483.

Valle JM, Estepa RM, Camacho RM, Estrada RC, et al. (2007). Endothelial dysfunction is related to insulin resistance and inflammatory biomarker levels in obese prepubertal children. Eur. J. Endocrinol. 156: 497-502.

Wake DJ, Strand M, Rask E, Westerbacka J, et al. (2007). Intra-adipose sex steroid metabolism and body fat distribution in idiopathic human obesity. Clin. Endocrinol. 66: 440-446.

Wheatcroft SB, Kearney MT, Shah AM, Ezzat VA, et al. (2007). IGF-binding protein-2 protects against the development of obesity and insulin resistance. Diabetes 56: 285-294. 